Address correspondence to: Paul R. Riley, University of Oxford, Department of Physiology, Anatomy and Genetics, Sherrington Building, South Parks Road, Oxford, OX1 3PT, United Kingdom. Phone: 44.0.1865.282366; Fax: 44.0.1865.272469; E-mail: paul.riley@dpag.ox.ac.uk.

1. Aurora AB, et al. Macrophages are required for neonatal heart regeneration. J Clin Invest. 2014; 124(3):1382-1392.

2. Porrello ER, et al. Transient regenerative potential of the neonatal mouse heart. Science. 2011; 331(6020):1078-1080.

3. Nahrendorf M, Pittet MJ, Swirski FK. Monocytes: protagonists of infarct inflammation and repair after myocardial infarction. Circulation. 2010; 121(22):2437-2445.

4. Nahrendorf $M$, et al. The healing myocardium sequentially mobilizes two monocyte subsets with divergent and complementary functions.J Exp Med. 2007;204(12):3037-3047.

5. Swirski FK, et al. Identification of splenic reservoir monocytes and their deployment to inflammatory sites. Science. 2009;325(5940):612-616
6. van Amerongen MJ, Harmsen MC, van Rooijen N, Petersen AH, van Luyn MJ. Macrophage depletion impairs wound healing and increases left ventricular remodeling after myocardial injury in mice. $A m$ I Pathol. 2007;170(3):818-829.

7. Roberts R, DeMello V, Sobel BE. Deleterious effects of methylprednisolone in patients with myocardial infarction. Circulation. 1976;53(3 suppl):I204-I206.

8 . Tsujioka $\mathrm{H}$, et al. Impact of heterogeneity of human peripheral blood monocyte subsets on myocardial salvage in patients with primary acute myocardial infarction. J Am Coll Cardiol. 2009;54(2):130-138.

9. Maekawa Y, et al. Prognostic significance of peripheral monocytosis after reperfused acute myocardial infarction: a possible role for left ventricular remodeling. J Am Coll Cardiol. 2002;39(2):241-246.

10. Panizzi $\mathrm{P}$, et al. Impaired infarct healing in atherosclerotic mice with Ly-6C(hi) monocytosis. J Am Coll Cardiol. 2010;55(15):1629-1638.

11. Frangogiannis NG. The immune system and cardiac repair. Pharmacol Res. 2008;58(2):88-111.

12. Lepilina A, et al. A dynamic epicardial injury response supports progenitor cell activity during zebrafish heart regeneration. Cell. 2006;127(3):607-619.

13. Itou J, et al. Migration of cardiomyocytes is essential for heart regeneration in zebrafish. Development. 2012;139(22):4133-4142.
14. van Rooijen N, van Nieuwmegen R. Elimination of phagocytic cells in the spleen after intravenous injection of liposome-encapsulated dichloromethylene diphosphonate. An enzyme-histochemical study. Cell Tissue Res. 1984;238(2):355-358.

15. Godwin JW, Pinto AR, Rosenthal NA. Macrophages are required for adult salamander limb regeneration. Proc Natl Acad Sci U S A. 2013;110(23):9415-9420.

16. Nahrendorf M, Swirski FK. Monocyte and macrophage heterogeneity in the heart. Circ Res. 2013; 112(12):1624-1633.

17. Ben-Mordechai T, et al. Macrophage subpopulations are essential for infarct repair with and without stem cell therapy. J Am Coll Cardiol. 2013; 62(20):1890-1901.

18. Jesty SA, et al. c-kit+ precursors support postinfarction myogenesis in the neonatal, but not adult, heart. Proc Natl Acad Sci U S A. 2012;109(33):13380-13385.

19. Tiemessen MM, Jagger AL, Evans HG, van Herwijnen MJ, John S, Taams LS. CD4+CD25+Foxp3+ regulatory $\mathrm{T}$ cells induce alternative activation of human monocytes/macrophages. Proc Natl Acad Sci US A. 2007;104(49):19446-19451.

20. Pinto AR, et al. An abundant tissue macrophage population in the adult murine heart with a distinct alternatively-activated macrophage profile. PLoS One. 2012;7(5):e36814.

\title{
Translational enhancers of EAAT2: therapeutic implications for neurodegenerative disease
}

\author{
Allison S. Limpert and Nicholas D.P. Cosford
}

Cell Death and Survival Networks Program, Sanford-Burnham Medical Research Institute, La Jolla, California, USA.

\begin{abstract}
Glutamate excitotoxicity contributes to the neuronal injury and death associated with many neurodegenerative diseases. The glutamate transporter EAAT2, which is primarily localized on astrocytic processes, facilitates glutamate clearance from synapses, thus preventing neuronal damage. In this issue of the JCI, Kong et al. characterize a compound that upregulates EAAT2 translation, thereby increasing glutamate uptake by glial cells. Furthermore, this strategy for alleviating excitotoxicity was found to be beneficial in mouse models of both amyotrophic lateral sclerosis (ALS) and epilepsy, suggesting that future development in this chemical series may lead to much-needed treatments for these disorders.
\end{abstract}

\section{Current therapies for neurodegenerative diseases offer limited relief}

Current treatments for many neurodegenerative diseases provide only very modest effects on disease progression and symptomology. While neurodegenerative disorders such as Parkinson's disease (PD), Alzheimer's disease (AD), and amyotrophic lateral sclerosis (ALS) affect an ever-growing number of individuals, our ability to

Conflict of interest: The authors have declared that no conflict of interest exists.

Citation for this article: $J$ Clin Invest. 2014;

124(3):964-967. doi:10.1172/JCI74608. treat these fatal illnesses is very limited. For example, ALS leads to the degeneration and death of motor neurons, causing muscle wasting, paralysis, and eventual death of affected individuals; however, the lone FDA-approved treatment currently available for this disorder only extends lifespan by a matter of months (1).

In this issue, Kong et al. propose a therapeutic strategy for the treatment of neurodegenerative disorders that targets the neuronal death and dysfunction caused by glutamate excitotoxicity (2). Damage to neurons caused by excess glutamate contributes to ALS and is a feature of many neurological conditions, including epilepsy, which is a group of disorders characterized by epileptic seizures resulting from aberrant cortical neuronal activity (3). Kong and colleagues characterize a compound, LDN/ OSU-0212320, that upregulates the translation of the glutamate transporter EAAT2 in astrocytes, thus increasing EAAT2 on glial cell surfaces and enhancing glutamate uptake (2). Previous work by this group entailed a small-molecule screen in PAEAAT2 cells, an EAAT2-expressing primary astrocyte line, for compounds that induce translational upregulation of EAAT2 (4). This led to a structure-activity relationship (SAR) study performed on the pyridazinebased series of small molecules identified in the aforementioned screen (5). The characterization of LDN/OSU-0212320 is the culmination of this work, demonstrating the efficacy of a representative compound from the pyridazine series in animal models of neuronal disorders.

Kong et al. demonstrate herein that within 2 hours of treatment, LDN/OSU-0212320 enhanced the localization of EAAT2 mRNA within polyribosome cell fractions, enhanced EAAT2 expression on the plasma 
A

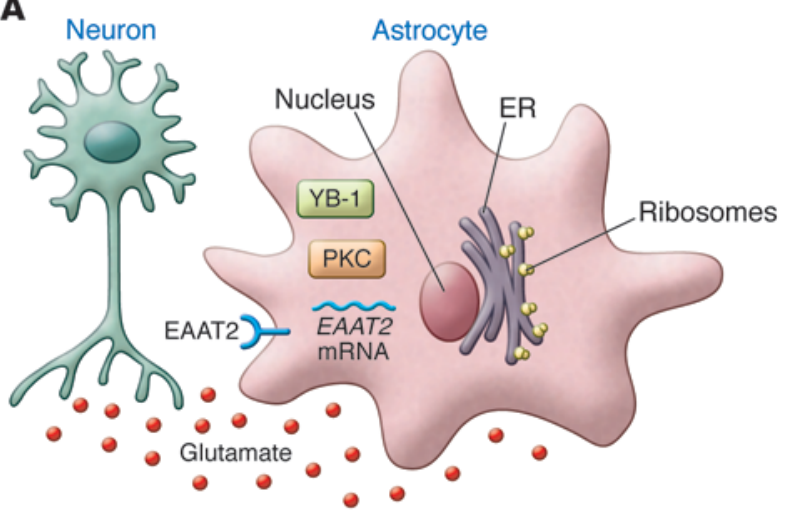

B

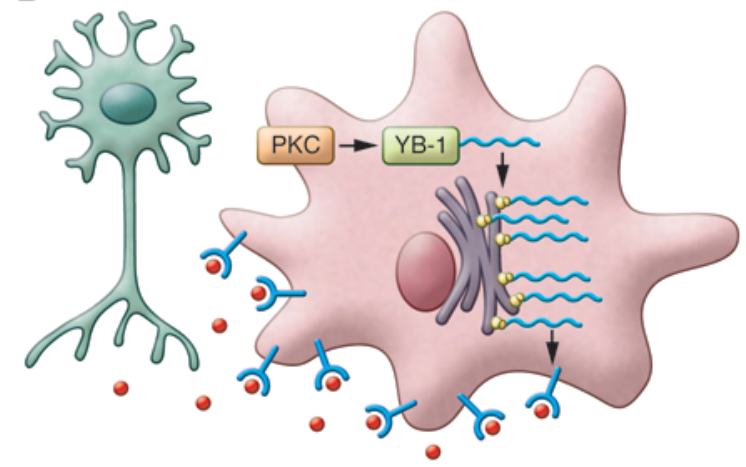

\section{Figure 1}

LDN/OSU-021230 upregulates EAAT2 expression in astrocytes. (A) In many neurodegenerative diseases, excess glutamate (red circles) causes damage to neurons (aqua), which can be mitigated by glutamate uptake by astrocytes (pink). (B) LDN/OSU-021230 increases glutamate uptake through the upregulation of EAAT2 receptors on astrocytic processes. Following LDN/OSU-021230 treatment, PKC becomes activated. This activation is required for phosphorylation of the translational regulator YB-1. LDN/OSU-021230 enhances the interaction of YB-1 with EAAT2 mRNA and upregulates the translation of EAAT2 protein. EAAT2 localizes to the plasma membrane, where it facilitates glutamate uptake by the cell, thus reducing glutamate excitotoxicity. membrane, and increased glutamate uptake in cultured astrocytes (2). Furthermore, in primary neuron-astrocyte cocultures, LDN/OSU-0212320 substantially protected neurons from glutamate-induced toxicity. LDN/OSU-0212320 demonstrated favorable pharmacokinetics (PKs) and localized to the brain of treated animals in appreciable concentrations following i.p. administration. These results, combined with the compound's good in vivo potency and low toxicity, open the possibility that LDN/OSU0212320 is a reasonable therapeutic option for the treatment of diseases that display glutamate-induced neurodegeneration.

\section{Enhanced glutamate transport in murine models of neurodegenerative disease}

Kong and colleagues tested LDN/OSU0212320 in two murine models of neurodegenerative disease: a model of chronic excitotoxicity and an acute excitotoxicity model (2). ALS is a disease characterized by loss of EAAT2 expression and chronic glutamate toxicity. SOD1(G93A) mice, an extensively used rodent model of ALS, were treated with LDN/OSU-021230 following symptom onset. LDN/OSU-0212320 treatment restored EAAT2 expression in the brain and resulted in both an exten- sion of lifespan and an enhancement of motor function. Additionally, LDN/OSU0212320 reduced mortality and spontaneous recurrent seizures in pilocarpineinduced status epilepticus mice, indicating that translational upregulation of EAAT2 may also be a viable treatment for epilepsy, which is also associated with acute glutamate toxicity.

The comprehensive study by Kong et al. on the potential for a pyridazine-based compound to increase EAAT2 translation and provide therapeutic efficacy in rodent models of neurodegenerative disease has compelling implications for ALS and epilepsy treatment (2). However, further studies will need to be performed to fully understand how these compounds may advance neurodegenerative therapies. While LDN/ OSU-021320-treated SOD1(G93A) mice exhibited pronounced symptom reduction and increased lifespan, it is important to note that many compounds that have demonstrated efficacy in this ALS model have had no significant effects in human trials. A pertinent example of such a compound is the $\beta$-lactam antibiotic ceftriaxone, which restores EAAT2 expression in SOD1(G93A) mice through increased Eaat2 transcription (6). Despite positive effects in SOD1(G93A) animals, phase III clinical trials of ceftriax- one in ALS patients were halted because the drug did not meet the predetermined efficacy criteria. Although SOD1(G93A) mice have historically been the most widely used murine ALS model, researchers are relying less and less on these animals as predictors of human outcomes.

Currently, the only FDA-approved treatment for ALS is riluzole, a compound that also reduces glutamate toxicity in vivo; however, riluzole confers few advantages to patients and only increases lifespan by several months (7). The minuscule benefits of riluzole, combined with the ineffectiveness of ceftriaxone in clinical trials, raises the question of whether a reduction in glutamate toxicity alone is an adequate treatment for ALS. As neurons in affected individuals suffer from a variety of biological insults, perhaps a reduction of excitotoxicity alone is not a realistic mechanism for therapeutic development. However, it will be imperative to determine whether drugs that reduce glutamate toxicity could be efficacious in ALS patients when used in combination with other treatments.

Additionally, Kong et al. provide data suggesting that translational enhancers of EAAT2 are advantageous for the treatment of epilepsy (2). Current anticonvulsants, predominantly $\mathrm{Na}^{+}$channel 
blockers, do not meet patients' needs, and approximately $30 \%$ of epilepsy disorders are reported to be drug resistant. Furthermore, many anticonvulsants have undesirable side effects, including sedation, ataxia, and weight loss or gain (8). Upregulation of glutamate transporters on astrocytes may be an effective treatment strategy, because excessive glutamatergic neurotransmission is believed to be one of the major pathological mechanisms of epilepsy. Notably, transgenic mice that lack EAAT2 have spontaneous epileptic activity (9). In addition, astrocytes contribute to epilepsy by releasing glutamate during seizure activity, implying that astrocytes induce irregular neuronal activity (10). These data suggest that a drug that enhances glutamate uptake by astrocytes may be a powerful tool for epilepsy treatment.

While LDN/OSU-0212320 did not have any measurable effect on acute seizure activity, Kong et al. demonstrated that treatment with this compound reduces neuronal death and mortality rates following seizure activity in mice (2). Importantly, LDN/OSU-0212320 substantially reduced recurrent seizures; therefore, this compound may attenuate the progression of this disease. Together, these data suggest that upregulation of EAAT2 expression and function may be an appropriate mechanism for the development of new therapeutics for patients with epilepsy. Kong and colleagues have provided evidence that initiation of EAAT2 translation is a drugable target that may allow for the treatment of drug-resistant forms of epilepsy. Additionally, LDN/OSU-0212320 appears to have a low side-effect profile, indicating that drugs developed around this target may have fewer adverse side effects than current anticonvulsants. A treatment strategy centered on astrocytes rather than neurons has exciting potential for epilepsy management.

\section{LDN/OSU-0212320 promotes EAAT2 translation via a YB-1-dependent mechanism}

Kong et al. also attempted to decipher the mechanism of action of LDN/OSU0212320 and demonstrated that this compound increases the association of EAAT2 mRNA with Y-box-binding protein 1 (YB1), a nucleic acid-binding protein that is important for translational regulation (2) (Figure 1). Knockdown of YB-1 with siRNA reduced the ability of LDN/OSU0212320 to upregulate EAAT2 expres- sion, indicating that $\mathrm{YB}-1$ is required for enhanced EAAT2-mediated glutamate uptake. Additionally, LDN/OSU-0212320 increased the phosphorylation of both YB-1 and the signaling effector protein kinase $\mathrm{C}$ (PKC). Further studies indicated that PKC activity is required, but not sufficient, for LDN/OSU-0212320 activation of EAAT2 translation. While these data provide some insight into the mechanism of action, the molecular target of LDN/ OSU-0212320 is still unknown. Due to the efficiency of biological systems, it seems unlikely that there is a molecular target with the sole function of regulating the translation of EAAT2 mRNA. While upregulation of actin mRNAs in polyribosomal fractions was not observed after LDN/OSU-0212320 treatment, the possibility that a series of other related mRNAs are recruited to polyribosomes following drug treatment cannot be eliminated. Additional investigations into the mechanism of action and molecular targets of this compound are necessary to predict its effects in human trials and to facilitate drug discovery efforts. Furthermore, future studies that include a screen to analyze protein upregulation following chronic compound treatment should be considered.

\section{Conclusions and future directions}

In conclusion, the demonstrated efficacy of an EAAT2 translational enhancer has enticing implications for the treatment of neurodegenerative diseases characterized by both chronic and acute glutamate toxicity. As a treatment for ALS, the study by Kong and colleagues (2) should be viewed with cautious optimism, because the preponderance of evidence suggests that due to the complex symptomology of ALS, a reduction of excess glutamate alone will be of little benefit to patients. However, elimination of excitotoxicity in combination with other treatments will likely improve patient outcomes. The development of compounds targeting EAAT2 translation for ALS therapy should be viewed in the context of a combinatorial treatment option, with discovery efforts focused on the development of compounds with druglike properties including PK profiles that favor use with other therapeutic agents.

In this study, Kong et al. also demonstrate that upregulation of glutamate transporters on astrocytes has pronounced benefits in mouse models of temporal lobe epilepsy (2). The fact that LDN/OSU-0212320 tar- gets translation rather than transcription of EAAT2 suggests that upregulation of this protein can be achieved quickly, providing protection from the acute excitotoxicity seen following status eptilepticus. While no mutations involving glutamatergic signaling have been identified in epilepsy patients (11), deficiencies in glutamate transport function have been documented (12); therefore, enhancing EAAT2 expression may be beneficial, and reduction of astrocytic glutamate may enhance the outcomes of conventional epilepsy treatments.

Finally, despite the substantial data included in this study, further investigation of the mechanism of action of these compounds is required. Understanding how these drugs directly target EAAT2 translation is important, especially for the prediction of drug interactions and side effects of this compound in patients.

\section{Acknowledgments}

This work was supported in part by the Department of Defense Amyotrophic Lateral Sclerosis Research Program under award number W81XWH-12-1-0373 (to N.D.P. Cosford). The views and opinions of and endorsements by the authors do not reflect those of the U.S. Army or the Department of Defense.

Address correspondence to: Nicholas D.P. Cosford, Sanford-Burnham Medical Research Institute, $10901 \mathrm{~N}$. Torrey Pines Rd., La Jolla, California 92037, USA. Phone: 858.795.5316; Fax: 858.795.5221; E-mail: ncosford@sanfordburnham.org.

1. Limpert AS, Mattmann ME, Cosford ND. Recent progress in the discovery of small molecules for the treatment of amyotrophic lateral sclerosis (ALS). Beilstein J Org Chem. 2013;9:717-732.

2. Kong Q, et al. Small-molecule activator of glutamate transporter EAAT2 translation provides neuroprotection. J Clin Invest. 2014;124(3):1255-1267.

3. Fisher RS, et al. Epileptic seizures and epilepsy: definitions proposed by the International League Against Epilepsy (ILAE) and the International Bureau for Epilepsy (IBE). Epilepsia. 2005; 46(4):470-472

4. Colton CK, et al. Identification of translational activators of glial glutamate transporter EAAT2 through cell-based high-throughput screening: an approach to prevent excitotoxicity. J Biomol Screen. 2010;15(6):653-662

5. Xing X, et al. Structure-activity relationship study of pyridazine derivatives as glutamate transporter EAAT2 activators. Bioorg Med Chem Lett. 2011; 21(19):5774-5777.

6. Berry JD, et al. Design and initial results of a multiphase randomized trial of ceftriaxone in amyotrophic lateral sclerosis. PLoS One. 2013;8(4):e61177.

7. Glicksman MA. The preclinical discovery of amyotrophic lateral sclerosis drugs. Expert Opin Drug Discov. 2011;6(11):1127-1138.

8. Moldrich RX, Chapman AG, De Sarro G, Meldrum 
BS. Glutamate metabotropic receptors as targets for drug therapy in epilepsy. Eur J Pharmacol. 2003;476(1-2):3-16.

9. Tanaka K, et al. Epilepsy and exacerbation of brain injury in mice lacking the glutamate transporter
GLT-1. Science. 1997;276(5319):1699-1702.

10. Tian GF, et al. An astrocytic basis of epilepsy. Nat Med. 2005;11(9):973-981.

11. Chapman AG. Glutamate and epilepsy. J Nutr. 2000;130(4S Suppl):1043S-1045S.
12. Kong Q, Takahashi K, Schulte D, Stouffer N, Lin $\mathrm{Y}$, Lin CL. Increased glial glutamate transporter EAAT2 expression reduces epileptogenic processes following pilocarpine-induced status epilepticus. Neurobiol Dis. 2012;47(2):145-154

\title{
Working toward immune tolerance in lung transplantation
}

\author{
Xinguo Jiang and Mark R. Nicolls
}

VA Palo Alto Health Care System, Palo Alto, California, USA. Stanford University, Stanford, California, USA.

\begin{abstract}
Long-term allograft survival is a major challenge facing solid organ transplantation. Recent studies have shown a negative correlation between infiltration of memory $T$ cells and allograft survival. Furthermore, blockade of leukocyte activation increases acceptance of transplanted organs, including heart, liver, and kidney. Lung allografts are associated with high rates of rejection, and therapies that increase acceptance of other transplanted organs have not translated into the lung. In this issue of the JCI, Krupnick and colleagues demonstrate in a murine model that lung allograft acceptance requires infiltration of a specific $T$ cell population into the graft. This study highlights the unique immunobiology of the lung and the complexity of lung transplant tolerance.
\end{abstract}

Lungs have evolved over the millennia by adapting to the continual bombardment of foreign antigens, inhaled from the breathable environment, with a unique capacity to dampen overly exuberant immune responses. Lung physiology is distinct compared with more sterile compartments in the body, like the peritoneum (1), which are not constantly immunologically challenged. Despite the propensity of the lungs to dampen the immune responses, achieving immune tolerance is a particularly elusive goal for pulmonary transplant recipients. While there are examples of successful steroid withdrawal in kidney and liver transplant recipients $(2,3)$, examples of liver transplant recipients able to come off all immunosuppression over time (4), and examples of successful tolerance induction protocols $(5,6)$, similar examples are vanishingly rare among lung transplant recipients. A compounding issue is that the bronchial artery circulation, which normally supports airways with high- $\mathrm{O}_{2}$ blood, is not restored at the time of transplantation. Lack of circulation

Conflict of interest: The authors have declared that no conflict of interest exists.

Citation for this article: J Clin Invest. 2014; 124(3):967-970. doi:10.1172/JCI74701. potentially decreases the ability of grafted lungs to fend off pathogens and cope with immune injury (7). The inherent antigenicity of the lung and its lymphatic drainage into bronchus-associated lymphoid tissue (BALT) $(8,9)$ appear to be coupled with an imperfect barrier defense against pathogen invasion. Together, these qualities ultimately render the lung particularly difficult to target for durable tolerance. To overcome these inherent obstacles to organ health, more information is clearly needed about the unique immunobiology of the transplanted lung.

In this issue of the JCI, Krupnick and colleagues (10) evaluated immune responses in murine orthotopic lung transplant recipients that were treated with costimulation blockade and specifically addressed how memory $\mathrm{T}$ lymphocytes contribute to the difficulty of achieving immune tolerance. Krupnick et al. discovered a population of memory $\mathrm{CD}^{+} \mathrm{T}$ cells that were paradoxically protective for the grafted lung through their regulatory function. These results further emphasize the unique complexity of pulmonary-specific transplant responses, which must be further evaluated if progress is to be made toward generating clinically important therapies to preserve protective immunity.

\section{Immune tolerance and acute rejection in lung transplantation}

Immune tolerance can be defined in several ways. In robust animal models, specific immune tolerance refers to an antigenspecific unresponsive immunologic state that preserves responsiveness to other antigens. At the other side of the spectrum is operational or "prope" (meaning "almost" or "near") tolerance, which describes an immune state in which only very lowlevel immunosuppression is required to maintain the health of the transplant (11). While specific immune tolerance is the goal of transplant researchers, prope tolerance appears to be a more realistic objective for lung transplant recipients, given the inherent challenges to allograft survival posed by constant environmental exposure, native immunogenicity, and compromised vascular supply.

While a prior study demonstrated that costimulation blockade of CD154/CD40 and $\mathrm{CD} 28 / \mathrm{B} 7$ interactions induces lung transplant acceptance, infection with the common bacterium Psendomonas aeruginosa abolished tolerance (12). Beyond frequent exposure to bacterial pathogens, lung transplant recipients may be especially vulnerable to aerosolized viruses that are able to directly trigger heterologous immunity against donor MHC $(13,14)$. Tolerance induction in the lung has been difficult to recapitulate in animal models of airway and lung transplantation. In murine models, combined therapy targeting CD154 and lymphocyte function-associated antigen 1 (LFA-1), promoted specific immune tolerance in pancreatic islet transplants but failed to do so with airway allografts $(15,16)$. Additionally, mixed chimerism generated through whole body irradiation, bone marrow transplantation, and a brief course of sev- 